BULLETIN OF THE

AMERICAN MATHEMATICAL SOCIETY

Volume 77, Number 3, May 1971

\title{
GENERATION OF EQUICONTINUOUS SEMIGROUPS BY HERMITIAN AND SECTORIAL OPERATORS. II
}

\author{
BY ROBERT T. MOORE ${ }^{1}$
}

Communicated by M. H. Protter, September 25, 1970

1. Introduction. This announcement concerns the topological aspects of the generation theory of equicontinuous semigroups and groups of operators on a complete complex locally convex space (lcs) $\mathfrak{X}$, and uses the recalibration theorem from [6] to relate these to the more geometrical aspects treated in [8] (with which the reader is assumed to be familiar). Perturbation techniques from [7], along with other devices, are used to develop applications to the theory of abstract heat equations and to the theory of distribution semigroups. Details will appear in [9].

2. Quasi-equicontinuous semigroups. The semigroups considered here generalize the contraction holomorphic semigroups $\mathrm{CH}(\Phi, \Gamma)$ on a complete complex lcs $\mathfrak{X}$ discussed in [8], where for, $0 \leqq \Phi \leqq \pi / 2$, $S_{\Phi}=\{z \in C:|\arg z| \leqq \Phi\}$ and $\Delta_{\Phi}=\{z \in C: \pi / 2+\Phi \leqq \arg z \leqq 3 \pi / 2-\Phi\}$.

Definition 1. Let $\omega \geqq 0$. Then a family $\left\{T_{z}: z \in S_{\Phi}\right\} \subset \mathcal{L}(\mathfrak{X})$ of continuous linear transformations is a quasi-equicontinuous holomorphic semigroup of type $\omega$, or is in $\mathrm{EH}(\Phi ; \omega)$ iff

(a) it satisfies the usual algebraic, continuity and holomorphy conditions as in Definition (1a) of [8], and

(b) the family $\left\{e^{-\omega z} T_{z}: z \in S_{\Phi}\right\}$ is equicontinuous in $\mathscr{L}(\mathfrak{X})$.

ExAmples. (1) If $\left\{T_{t}: t \in[0, \infty)\right\}$ is a classical $C_{0}$ semigroup on a $B$-space [3], and $\omega>\omega_{0}=\lim \left\{\left(t^{-1} \log \left\|T_{t}\right\|\right): t \rightarrow \infty\right\}$, then $\left\|T_{t}\right\| \leqq M e^{\omega t}$ for suitable $M$ and the semigroup is in $\operatorname{EH}(0 ; \omega)$ since operator-normbounded sets are equicontinuous. Similarly, every semigroup in Hille's class $H(-\Psi, \Psi)$ on a $B$-space $[3]$ is in $\operatorname{EH}(\Phi ; \omega(\Phi))$ for every $\Phi<\Psi$ and suitable $\omega(\Phi)$.

(2) Every $\mathrm{CH}(\Phi, \Gamma)$ semigroup from $[8]$ is in $\mathrm{EH}(\Phi ; 0)$.

(3) Every equicontinuous $C_{0}$ semigroup as in Yosida [10] is in

AMS 1969 subject classifications. Primary 4750, 4748; Secondary 4601.

Key words and phrases. $C_{0}$ equicontinuous semigroups, quasi-equicontinuous holomorphic semigroup, exponential distribution semigroup, contraction semigroup, infinitesimal generator, sectorial operator, hermitian operator, analytic continuation of semigroups, abstract Laplacian, abstract heat equation.

1 The author gratefully acknowledges partial support of this research by the National Science Foundation through NSF GP 12548.

Copyright (1) 1971, American Mathematical Society 
$\mathrm{EH}(0 ; 0) ;$ Miyadera $[5]$ discusses $\mathrm{EH}(0 ; \omega)$ semigroups on Fréchet spaces.

As in Definition 2 of [8], the (infinitesimal) generator $A$ of an $\mathrm{EH}(\Phi ; \omega)$ semigroup is defined, wherever the limit exists, by

$$
A u=\lim \left\{t^{-1}\left(T_{t} u-u\right): t \rightarrow 0 \text { in }[0, \infty] \subset S_{\Phi}\right\}
$$

and the semigroup is smooth iff this limit exists for all $u \in \mathfrak{X}$.

THEOREM 1. Let $0 \leqq \Phi \leqq \pi / 2$ and let $\omega \geqq 0$. The following conditions on an operator $A$ are equivalent.

(a) The domain $D(A)$ is dense in $\mathfrak{X}, A$ is closed, the finite spectrum of $A, \sigma_{F}(A) \subset \Delta_{\Phi}+\omega\left(c f\right.$. [6]) and if $d_{\lambda}=\operatorname{dist}\left(\lambda, \Delta_{\Phi}+\omega\right)$ then

$$
\left\{\left[d_{\lambda}(\lambda-A)^{-1}\right]^{n}: d_{\lambda}>0, n=0,1, \cdots\right\}
$$

is equicontinuous.

(b) The operator $A$ is closed and densely defined, and $A-\omega$ is $\Phi$-sectorial with respect to some calibration $\Gamma$ for $\mathfrak{X}$.

(c) There exists a calibration $\Gamma$ for $\mathfrak{X}$ such that $A-\omega$ generates a semigroup $\left\{T_{z}^{\omega}=e^{-\omega z} T_{z}: z \in S_{\Phi}\right\}$ in $\mathrm{CH}(\Phi, \Gamma)$.

(d) The operator $A$ generates an $\mathrm{EH}(\Phi ; \omega)$ semigroup $\left\{T_{z}: z \in S_{\Phi}\right\}$.

Proof scheme. All implications in $(\mathrm{a}) \Leftrightarrow(\mathrm{b}) \Leftrightarrow(\mathrm{c}) \Leftrightarrow(\mathrm{d})$ are routine from [6], [7], and [8], with the exception of $(b) \Rightarrow(a)$, which requires recalibration by an equicontinuous simply closed convex balanced semigroup $S$ of operators generated by the $d_{\lambda}(\lambda-A)^{-1}$ for $\lambda \in S_{\Phi}$.

Similarly, a group $\left\{T_{t}: t \in R\right\}$ is in $E C_{0}(\omega)$ iff it is $C_{0}$ as in Definition 4 of $[8]$ and $\left\{e^{-\omega|t|} T_{t}: t \in R\right\}$ is equicontinuous. The analog of Theorem 1 is straightforward, with $\sigma_{F}(A)$ and $W\left(A, \Lambda_{*}\right)$ contained in the strip $\{z:|\operatorname{Re}(z)| \leqq \omega\}$ in (a) and (b) respectively. The case $\omega=0$ is of primary interest.

THEOREM 2. If $A$ is as in Theorem 1, the following are equivalent.

(a) The finite spectrum of closed, densely defined $A$ is pure imaginary, and $\left\{\left[|\operatorname{Re}(\lambda)|(\lambda-A)^{-1}\right]^{n}: n=0,1, \cdots, \operatorname{Re}(\lambda) \neq 0\right\}$ is equicontinuous.

(b) The operator iA is closed, densely defined, and hermitian with respect to a suitable calibration $\Gamma$.

(c) A generates a generalized unitary group $\left\{T_{t}: t \in R\right\}$ in $R C_{0}(\Gamma)$ for some suitable $\Gamma$.

(d) $A$ generates an $E C_{0}(0)$ group $\left\{T_{t}: t \in R\right\}$.

3. Semigroups on Banach sub-and-super-spaces. We show here that the properties of $\operatorname{EH}(\Phi ; \omega)$ semigroups are entirely determined by their natural classical actions on certain appropriate classes of 
Banach subspaces and super-spaces of the given lcs $\mathfrak{X}$. To simplify the discussion, suppose that $\mathfrak{X}$ admits a continuous norm, hence a calibration $\Gamma$ consisting of norms. (Recalibration preserves this property.)

Then the super-spaces are formed by completing $\mathfrak{X}$ with respect to a particular norm $p \in \Gamma$ to obtain a $B$-space $\mathfrak{X}_{p}$.

TheOREM 3. Let $\left\{T_{z}: z \in S_{\Phi}\right\}$ be an operator-valued function. Then it is an $\mathrm{EH}(\Phi ; \omega)$ semigroup iff there exists a norm-calibration $\Gamma$ for $\mathfrak{X}$ such that every $T_{z}$ has an extension-by-continuity $T_{z}^{p} \in \Theta\left(\mathfrak{X}_{p}\right)$ and $\left\{e^{-\omega z} T_{z}^{p}: z \in S_{\Phi}\right\}$ forms a $\mathrm{CH}(\Phi,\{p\})$ semigroup on $\mathfrak{X}_{p}$ for all $p \in \Gamma$.

The subspaces are defined in terms of functions $F: \Gamma \rightarrow(0, \infty)$, letting $\|u\|_{F}=\left\{\sup F(p)^{-1} p(u): p \in \Gamma\right\} \in[0, \infty)$ and

$$
\mathfrak{X}_{F}=\left\{u \in \mathfrak{X}:\|u\|_{F}<\infty\right\}
$$

with bounded unit ball $\mathfrak{B}_{F}=\left\{u \in \mathfrak{X}:\|u\|_{F} \leqq 1\right\}$. Then

$$
\mathfrak{X}=\bigcup\left\{\mathfrak{X}_{F}: F: \Gamma \rightarrow(0, \infty)\right\}=\bigcup\left\{\mathfrak{B}_{F}: F: \Gamma \rightarrow(0, \infty)\right\} .
$$

THEOREM 4. Let $\left\{T_{z}: z \in S_{\Phi}\right\}$ be an operator-valued function. Then it is an $\mathrm{EH}(\Phi ; \omega)$ semigroup iff there exists a norm-calibration $\Gamma$ for $\mathfrak{X}$ satisfying the two conditions:

(1) every $T_{z}$ leaves every $\mathfrak{X}_{F}$ invariant and restricts to a $T_{z}^{F} \in \Theta\left(\mathfrak{X}_{F}\right)$ with $\left\|e^{-\omega_{z}} T_{2}^{F}\right\|_{F} \leqq 1$, and

(2) for some $\|\cdot\|_{F}$-closed, $\Gamma$-dense subspace $\mathfrak{Y}_{F} \subset \mathfrak{X}_{F}$ invariant under the $T_{z}^{F}$, the restricted $\left\{e^{-\omega z} T_{z}^{F}: z \in S_{\Phi}\right\} \subset \otimes\left(Y_{F}\right)$ is a $\mathrm{CH}\left(\Phi, \quad\left\{\|\cdot\|_{F}\right\}\right)$ semigroup.

REMARK. It is not yet clear whether $\Gamma$ can be chosen so that $\left\{e^{-\omega z} T_{2}^{F}: z \in S_{\Phi}\right\}$ is in $\operatorname{CH}\left(\Phi,\left\{\|\cdot\|_{F}\right\}\right)$ on all of every $\mathfrak{X}_{F}$, although it is necessarily operator-norm holomorphic there on $\operatorname{int}\left(S_{\Phi}\right)$.

The inevitable variants of these results are also true for groups.

4. Exponential distribution semigroups. Lions [4] has defined, and proved a "Hille-Yosida theorem" for, a generalized class of exponential distribution semigroups of type $\leqq \omega$, (or $\operatorname{ED}(0 ; \omega)$ semigroups) on a $B$-space $\mathfrak{X}$. These are $B(\mathfrak{X})$-valued distributions (where $B(\mathfrak{X})$ carries the norm topology) which restrict to algebra homomorphisms into $B(\mathfrak{X})$ from the convolution algebra $D_{0}$ of $C^{\infty}$ functions $\varphi$ compactly supported in $[0, \infty)$, forming $\left\{T(\varphi): \varphi \in D_{0}\right\}$. In addition to technical hypotheses governing behavior near 0 , one assumes that the map $\varphi \rightarrow$ " $T\left(e^{-\omega t} \varphi(t)\right)$ " extends to a tempered distribution. These arise classically if $\left\{T_{t}: t \in[0, \infty)\right\}$ is a semigroup homomorphism which is strongly continuous on the open set $(0, \infty)$ and satisfies the integra- 
bility condition at $0: \int_{0}^{1}\left\|T_{t} u\right\| d t<\infty$ for all $u \in X$, as in the HillePhillips semigroups of class $(0, A)[3]$. Then $\left\{T(\varphi): \varphi \in D_{0}\right\}$ is defined by integration for $\varphi \in \mathscr{D}_{0}$ and $u \in \mathfrak{X}$ by

$$
T(\varphi) u=\int_{0}^{\infty} \varphi(t) T_{t} u d t
$$

A classical theorem of Feller [1], relating these more general classical semigroups to those in $\mathrm{EH}(0 ; \omega)$, can be generalized using Theorems 3 and 4 along with results due to Fujiwara [2].

THEOREM 5. If $\left\{T(\varphi): \varphi \in D_{0}\right\}$ is in $\operatorname{ED}(0 ; \omega)$, then there exists a dense subspace $\mathfrak{Y} \subset \mathfrak{X}$, which is a $B$-space with respect to a stronger norm $\|\cdot\|_{c} \geqq\|\cdot\|$, and a classical $C_{0}$ semigroup $\left\{T_{t}: t \in[0, \infty)\right\}$ on $\left(\mathfrak{Y},\|\cdot\|_{c}\right)$ such that for all $u \in \mathfrak{Y}$ and $\varphi \in \mathscr{D}_{0}$, (2) holds and characterizes $\left\{T(\varphi): \varphi \in D_{0}\right\}$ uniquely.

ReMARKs. (1) Either $\mathfrak{Y}$ is first-category in $\mathfrak{X}$, or $\mathfrak{Y}=\mathfrak{X}$ as topological vector spaces, and in the latter case the $\operatorname{ED}(0 ; \omega)$ semigroup is exactly the integrated form (2) of $\left\{T_{t}: t \in[0, \infty)\right\}$.

(2) "Holomorphic" exponential distribution semigroups have been defined on sectors $S_{\Phi}$ for $0<\Phi \leqq \pi / 2$ in the work of Fujiwara and others [2], leading naturally to a class of $\operatorname{ED}(\Phi ; \omega)$ semigroups to which Theorem 5 easily extends.

Generalization. If $B(\mathfrak{X})$ in the definition of an $\operatorname{ED}(\Phi ; \omega)$ semigroup is replaced by $\mathcal{F}_{\Gamma}(X)$ on a general locally convex space, the entire Lions theory admits a reasonable generalization to this setting. A satisfactory $\Gamma$-independent theory has not yet been obtained.

5. Yosida's analytic continuation theorem. The recalibration theorem can be used to clarify, strengthen, and trivially generalize an important theorem of Yosida (cf. §IX.10 of [10]) concerning continuation of an $\mathrm{EH}(0 ; 0)$ semigroup from $[0, \infty)$ to a suitable $S_{\Phi}$ for $\Phi>0$ in order to obtain an $\mathrm{EH}(\Phi ; \omega)$ semigroup.

Theorem 6. Let $\left\{T_{t}: t \in[0, \infty)\right\}$ be an $\mathrm{EH}(0 ; \omega)$ semigroup with generator $A$. Then (a), (b) and (c) are equivalent, and imply (d).

(a) For all $t>0, T_{t} \mathfrak{X} \subset D(A)$, and there exists a $c>0$ such that $\left\{\left[c t(A-\omega) e^{-\omega t} T_{t}\right]^{n}: 0<t \leqq 1\right.$ and $\left.n=0,1, \cdots\right\}$ is an equicontinuous family of operators on $\mathfrak{X}$.

(b) For all $t>0, T_{t} \mathfrak{X} \subset D(A)$ and there exists a $c>0$ and a calibration $\Gamma$ for $\mathfrak{X}$ such that $A T_{t} \in \mathcal{F}_{\Gamma}(\mathfrak{X})$ and $\left\|(A-\omega) T_{t}\right\|_{\Gamma} \leqq\left(c t e^{-\omega t}\right)^{-1}$ for all $0<t \leqq 1$.

(c) For some calibration $\Gamma$ and $c>0$ the series in the formula 


$$
T_{z}=e^{\omega x} \sum\left\{\frac{(z-t)^{n}}{n !}\left[(A-\omega) e^{-\omega t} T_{t}\right]^{n}: n=0,1, \cdots\right\}
$$

converges in the $\|\cdot\|_{\mathrm{r}}$ sense whenever $|z-t|<c t e^{-1}$.

(d) For some constant $c>0$ and any $\Phi<\sin ^{-1}\left(c e^{-1}\right)$, there exists an $\omega(\Phi) \geqq \omega$ such that $A$ generates an $\mathrm{EH}(\Phi ; \omega(\Phi))$ semigroup described by (3).

REMARKs. (1) If $c>e$, then $A$ is a finite operator, the power series $\exp (z A)=\sum\left((z A)^{n} / n !\right)$ yields an entire analytic continuation of $\left\{T_{t}: t \in[0, \infty)\right\}$, and for any $\omega>r_{E}(A)$ (the spectral radius) the extension is in $\mathrm{EH}(\pi / 2 ; \omega)$.

(2) If the conditions in (a) or (b) hold for all $0<t<\infty$, rather than just for $0<t \leqq 1$, then the formula (3) defines a semigroup extendable by limits to $S_{\Phi}$ with $\Phi=\sin ^{-1}\left(c e^{-1}\right)$ to yield a semigroup in $\operatorname{EH}(\Phi ; \omega)$ with the same growth rate on $S_{\Phi}$ as on $[0, \infty)$. For $c=e$ and $\omega=0$, this gives a condition for holomorphic continuations equicontinuous in the closed half-plane. (This remark is due in large part to B. Dembart (private communication).)

(3) The methods of Yosida (loc. cit.) can be used to prove that (a)-(c) are also equivalent to a resolvent condition:

(e) For any $\epsilon>0$ there exists a constant $d_{\epsilon}>0$ such that $\left.\left[d_{\epsilon} \lambda(\lambda+\omega-A)^{-1}\right]^{n}: \lambda>0, n=0,1, \cdots\right\}$ is equicontinuous. Theorem 6 can be used to prove that this in fact is equivalent to the superficially much stronger resolvent condition:

(f) For some calibration $\Gamma, \Phi>0$ and $\omega(\Phi) \geqq \omega, \sigma_{\Gamma}(A) \subset \Delta_{\Phi}+\omega(\Phi)$ and if $d_{\lambda}=\operatorname{dist}\left(\lambda, \Delta_{\Phi}+\omega(\Phi)\right)$ then $\left\|(\lambda-A)^{-1}\right\| \leqq d_{\lambda}^{-1}$.

6. Integration of certain abstract heat equations. Suppose that $\left\{T_{t}^{(j)}: t \in R\right\}, 1 \leqq j \leqq n$, is a collection of $n$ smooth $E C_{0}$ (0) groups on $\mathfrak{X}$, with continuous generators $A_{j} \in \mathfrak{L}(\mathfrak{X})$. Then we define an abstract Laplacian operator by

$$
L=\sum\left\{A_{j}^{2}: 1 \leqq j \leqq n\right\}
$$

in $\mathcal{L}(\mathfrak{X})$, and seek solutions to the abstract heat equation for $\{u(t): t \in[0, \infty)\}:$

$$
\frac{d}{d t} u(t)=L u(t), \quad t \in(0, \infty) ; u(0)=u_{0} \in \mathfrak{X} .
$$

Utilizing Yosida's ideas on "holomorphic Markov processes" [11], the following partial results have been obtained.

TheOREM 7. Suppose the multiplicative group in $\mathfrak{L}(\mathfrak{X})$ generated by 
all of the operators $\left\{T_{t}^{())}: t \in R\right.$ and $\left.1 \leqq j \leqq n\right\}$ is equicontinuous. Then $L$ generates a smooth $\mathrm{EH}(0 ; 0)$ semigroup $\left\{P_{t}: t \in[0, \infty)\right\}$ such that for every initial condition $u_{0} \in \mathfrak{X}, u(t)=P_{t} u_{0}$ solves (5).

Theorem 8. Suppose $T_{s}^{(f)} T_{t}^{(k)}=T_{t}^{(k)} T_{s}^{(f)}$ for all $s, t \in R$ and $j, k$ between 1 and $n$. Then for every $0 \leqq \Phi \leqq \pi / 2$ there exists an $\omega(\Phi)$ such that $L$ generates an $\mathrm{EH}(\Phi ; \omega(\Phi))$ semigroup $\left\{P_{z}: z \in S_{\Phi}\right\}$ which is equicontinuous on the real axis and such that $u(t)=P_{t} u_{0}$ solves (5) as above.

Remark. Analytic continuation off the axis in the setting of Theorem 7 is likely, but has not conclusively been established.

ExAmple. The Laplacian on the $n$-torus discussed in Example 2 of [8] is typical of the generalized Laplacians treated above. (Notice that both theorems apply.) This gives an entirely abstract, operatortheoretic integration of the heat equation on the $n$-torus which avoids the "deficiency" problems and ellipticity techniques of the $L^{2}$ theory.

\section{REFERENCES}

1. W. K. Feller, On the generation of unbounded semigroups of bounded linear operators, Ann. of Math. (2) 58 (1953), 166-174. MR 14, 1093.

2. D. Fujiwara, A characterization of exponential distribution semi-groups, J. Math. Soc. Japan 18 (1966), 265-274. MR 33 \#6420.

3. E. Hille and R. S. Phillips, Functional analysis and semi-groups, Amer. Math. Soc. Colloq. Pub., vol. 31, Amer. Math. Soc., Providence, R. I., 1957. MR 19, 664.

4. J. L. Lions, Les semi groupes distributions, Portugal. Math. 19 (1960), 141-164. MR $26 \# 611$.

5. I. Miyadera, Semi-groups of operators in Fréchet space and applications to partial differential equations, Tôhoku Math. J. (2) 11 (1959), 162-183. MR 21 \#7445.

6. R. T. Moore, Banach algebras of operators on locally convex spaces, Bull. Amer. Math. Soc. 75 (1969), 68-73. MR 38 \#5018.

7. - Adjoints, numerical ranges, and spectra of operators on locally convex space, Bull. Amer. Math. Soc. 75 (1969), 85-90. MR 39 \#805.

8. - Generation of equicontinuous semigroups by Hermitian and sectorial operators. I, Bull. Amer. Math. Soc. 77 (1971), 224-229.

9. - Operator theory on locally convex spaces. I. Banach algebras, states, and numerical ranges (in prep.).

10. K. Yosida, Functional analysis, 2nd ed., Die Grundlehren der math. Wissenschaften, Band 123, Springer-Verlag, New York, 1968. MR 39 \#741.

11. - On holomorphic Markov processes, Proc. Japan. Acad. 42 (1966), 313-317. MR 34 \#6864.

University of Washington, Seattle, Washington 98105 